\title{
THE GENUS OF PERIODIC LINKS WITH RATIONAL QUOTIENTS
}

\author{
SANG YOUL LEE and MYOUNGSOO SEO ${ }^{\bowtie}$
}

(Received 12 May 2008)

\begin{abstract}
In this paper, we prove that if $K$ is any periodic link in $S^{3}$ whose quotient link is a 2-bridge link, then one half of the degree of the reduced Alexander polynomial, the minimal genus, the free genus and the canonical genus of $K$ are all the same. We also give criteria to determine whether a given periodic link has a 2-bridge link quotient and some properties of this kind of periodic link.
\end{abstract}

2000 Mathematics subject classification: primary 57M25.

Keywords and phrases: periodic link, genus, 2-bridge link, homogeneous link.

\section{Introduction}

A link $L$ in $S^{3}$ is called a $p$-periodic link ( $p \geq 2$ an integer) if there exists an orientation-preserving auto-homeomorphism $h$ of $S^{3}$ such that $h(L)=L, h$ is of order $p$ and the set $\operatorname{Fix}(h)$ of fixed points of $h$ is a circle disjoint from $L$. In this case, the link $L /\langle h\rangle \cup \operatorname{Fix}(h)$ in the orbit space $S^{3} /\langle h\rangle \cong S^{3}$ is called the quotient link of $L$ and $L /\langle h\rangle$ is called the factor link of $L$. Let $K$ be an oriented link in $S^{3}$ and $U$ an oriented trivial knot with $K \cap U=\emptyset$. For any integer $p \geq 2$, let $\phi_{U}^{p}: \Sigma^{3} \rightarrow S^{3}$ be a $p$ fold branched cyclic covering branched along $U$. Then $\Sigma^{3}$ is homeomorphic to $S^{3}$ and $\left(\phi_{U}^{p}\right)^{-1}(K)$ is a $p$-periodic link in $\Sigma^{3}$ with $L=K \cup U$ as its quotient link. We give an orientation to $\left(\phi_{U}^{p}\right)^{-1}(K)$ induced by the orientation of $K$. Note that any periodic knot or link in $S^{3}$ arises in this manner.

It is known [5, 9] that a 2-bridge link of 2-components can be represented by a Conway normal form $C\left(2, n_{1},-2, n_{2}, \ldots, n_{r},(-1)^{r} 2\right)$, which is equivalent to the diagram shown in Figure 1. Throughout this paper, the 2-bridge link $L$ in $S^{3}$ represented by Conway normal form $C\left(2, n_{1},-2, n_{2}, \ldots, n_{r},(-1)^{r} 2\right)$ with an orientation as shown in Figure 1 will be denoted by $L=\vec{C}\left[\left[n_{1}, n_{2}, \ldots, n_{r}\right]\right]$. In Figure 1, each tangle labeled with an integer $n_{i}(1 \leq i \leq r)$ denotes a 2-tangle as shown in Figure 2.

(C) 2009 Australian Mathematical Society 0004-9727/2009 \$16.00 


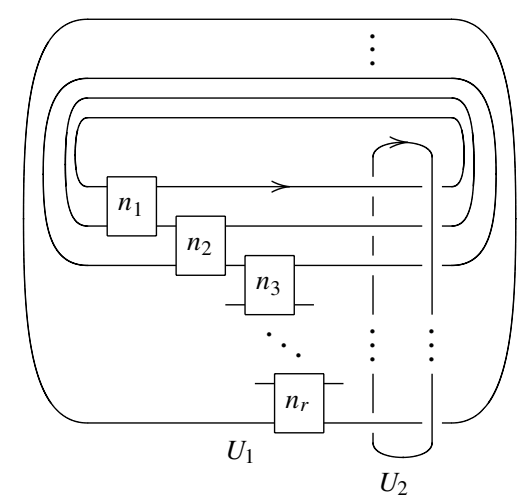

FIGURE 1. Diagram of a 2-bridge link of 2-components.

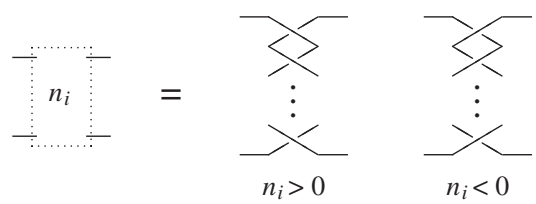

FIGURE 2. Tangle labeled with $n_{i}$.

A link $\widetilde{L}$ in $S^{3}$ is called a p-periodic link with rational quotient if there exists a 2-bridge link $L=U_{1} \cup U_{2}$ in $S^{3}$ such that $\widetilde{L}$ is equivalent to the preimage $\left(\phi_{U_{2}}^{p}\right)^{-1}\left(U_{1}\right)$ of the component $U_{1}$ of $L$ by a $p$-fold branched cyclic covering $\phi_{U_{2}}^{p}$ : $\Sigma^{3} \rightarrow S^{3}$ branched along the component $U_{2}$ of $L$. Note that each component $U_{1}$ and $U_{2}$ of the 2-bridge link $L$ is a trivial knot and they can be interchanged by an orientation-preserving homeomorphism of $S^{3}$ [7]. This guarantees that $\left(\phi_{U_{2}}^{p}\right)^{-1}\left(U_{1}\right)$ is equivalent to $\left(\phi_{U_{1}}^{p}\right)^{-1}\left(U_{2}\right)$. Let $L=\vec{C}\left[\left[n_{1}, n_{2}, \ldots, n_{r}\right]\right]$ be an oriented 2-bridge link as shown in Figure 1. Then the diagram $D^{(p)}$ shown in Figure 3 is called the canonical oriented $p$-periodic diagram of the oriented $p$-periodic link with rational quotient $L$. In what follows, we denote the oriented $p$-periodic link by $L^{(p)}$ or $\vec{C}\left[\left[n_{1}, n_{2}, \ldots, n_{r}\right]\right]^{(p)}$. Note that any $p$-periodic link with rational quotient can be represented by $\vec{C}\left[\left[n_{1}, n_{2}, \ldots, n_{r}\right]\right]^{(p)}$ for some nonzero integers $n_{1}, n_{2}, \ldots, n_{r}$. For more details, we refer the interested reader to $[6,9]$.

Frankel and Pontrjagin [4] and Seifert [17] introduced a method to construct an orientable closed surface having a given knot as its boundary. Seifert [17] defined the (minimal) genus $g(K)$ of a knot $K$ as the minimum of the genera of orientable closed surfaces having $K$ as a boundary and proved that

$$
\frac{1}{2} \operatorname{deg} \Delta_{K}(t) \leq g(K),
$$

where $\operatorname{deg} \Delta_{K}(t)$ is the degree of the Alexander polynomial $\Delta_{K}(t)$ of $K$. If $K$ is a torus knot, then the equality in (1.1) holds, but there are also cases where the equality 


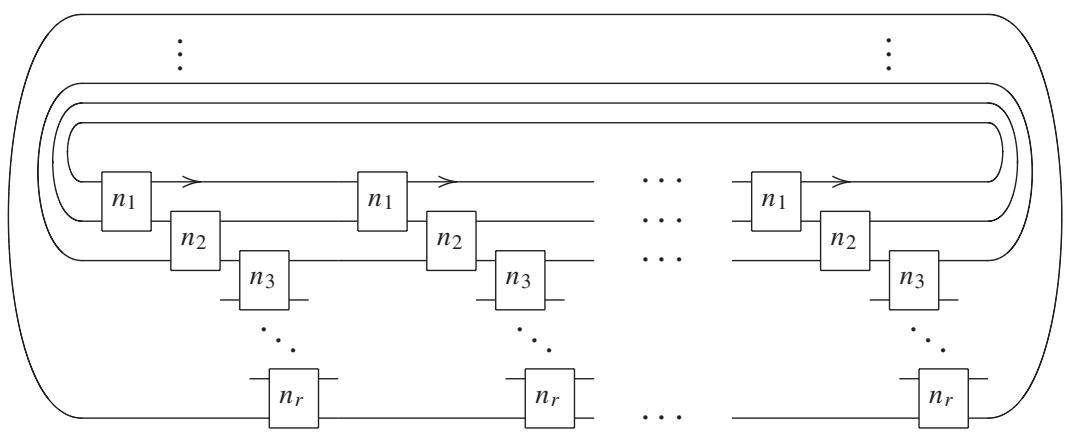

FIGURE 3. Canonical oriented $p$-periodic diagram.

does not hold. In fact, the trivial knot is the only knot with genus zero and there are many nontrivial knots whose Alexander polynomials are equal to one. The canonical genus $g_{c}(K)$ of a knot $K$ is defined to be the minimum of the genera of orientable closed surfaces obtained by Seifert's construction (algorithm) [17]. Note that Seifert's algorithm applied to a knot or link diagram might not produce a minimal genus Seifert surface. In addition, the free genus $g_{f}(K)$ and slice genus $g_{s}(K)$ of a knot $K$ have been introduced. It is easily seen that any canonical Seifert surface is free and, in general, the following inequality holds:

$$
g_{s}(K) \leq g(K) \leq g_{f}(K) \leq g_{c}(K) .
$$

Up to now, many authors have constructed knots and links for which this inequality is strict; see, for example, [15, 19]. On the other hand, Murasugi [10,11] proved that if $K$ is an alternating knot, then the equality in (1.1) holds and $g(K)=g_{c}(K)$ in (1.2).

In this paper, we prove that if $K$ is any $p$-periodic knot with rational quotient $\vec{C}\left[\left[n_{1}, n_{2}, \ldots, n_{r}\right]\right]$, then the equality in (1.1) holds and $g(K)=g_{f}(K)=g_{c}(K)=$ $r(p-1) / 2$ (see Theorem 3.2). Indeed, we study this for the class of all periodic knots and links with rational quotients. It should be noted that the class of all periodic knots and links with rational quotients includes all 2-bridge knots and links [6] and all torus knots and links, and also contains some nonalternating knots and links. We also investigate some other properties of knots and links contained in this interesting class and we give criteria to determine whether a given periodic link has a rational quotient or not (see Corollaries 2.4 and 3.3).

In Section 2, we determine the degree of the reduced Alexander polynomial of periodic links with rational quotients (see Theorem 2.3). In Section 3, we determine the minimal genus and prove that the free genus, the canonical genus of this kind of periodic link are all equal to the minimal genus, and that the orientable closed surface obtained from the canonical periodic diagram $D^{(p)}$ as shown in Figure 3 by Seifert's construction is a minimal genus surface (see Theorem 3.2). We also give a certain class of 3-periodic links with rational quotients for which the equality in (1.2) holds 
(see Theorem 3.5). In Section 4, we show that these periodic links are all homogeneous and give some related properties (see Theorem 4.1).

\section{The degree of the reduced Alexander polynomial}

We begin by reviewing a recursive formula for the Alexander polynomials of 2-bridge links given by the authors [9]. For a given integer $n$, let $R_{a}(t ; n), R_{b}(t ; n)$, $L_{a}(t ; n), L_{b}(t ; n)$ and $T_{n}(t)$ be Laurent polynomials in $\mathbb{Z}\left[t^{ \pm 1}\right]$ defined by

$$
\begin{aligned}
R_{a}(t ; n) & =(-1)^{n} t^{-\left(1+(-1)^{n}\right) / 2}\left\{\left\lfloor\frac{n}{2}\right\rfloor t-\left(\left\lfloor\frac{n}{2}\right\rfloor+\frac{1-(-1)^{n}}{2}\right)\right\}, \\
R_{b}(t ; n) & =(-1)^{n+1} t^{-\left(1+(-1)^{n}\right) / 2}\left\{\left\lfloor\frac{n}{2}\right\rfloor t-\left(\left\lfloor\frac{n}{2}\right\rfloor-\frac{1+(-1)^{n}}{2}\right)\right\}, \\
L_{a}(t ; n) & =(-1)^{n} t^{-\left(1+(-1)^{n}\right) / 2}\left\{\left(\left\lfloor\frac{n}{2}\right\rfloor+\frac{1-(-1)^{n}}{2}\right) t-\left(\left\lfloor\frac{n}{2}\right\rfloor+1\right)\right\}, \\
L_{b}(t ; n) & =(-1)^{n+1} t^{-\left(1+(-1)^{n}\right) / 2}\left\{\left(\left\lfloor\frac{n}{2}\right\rfloor+\frac{1-(-1)^{n}}{2}\right) t-\left\lfloor\frac{n}{2}\right\rfloor\right\}, \\
T_{n}(t) & =(-1)^{n+1} t^{-\left(1+(-1)^{n}\right) / 2},
\end{aligned}
$$

where $\lfloor x\rfloor$ is the largest integer less than or equal to $x$. Let $n_{1}, n_{2}, \ldots, n_{r}$ be given integers $(r \geq 1)$. For each $k=1,2, \ldots, r$, we put

$$
\varepsilon_{0}=1, \quad \varepsilon_{k}=(-1)^{n_{1}+\cdots+n_{k}+k}, \quad \mathcal{T}^{k}(t)=T_{n_{k}}\left(t^{\varepsilon_{k-1}}\right)
$$

and

$$
\begin{array}{rlrl}
\mathcal{R}_{a}^{k}(t) & =R_{a}\left(t^{\varepsilon_{k-1}} ; n_{k}\right), & \mathcal{R}_{b}^{k}(t) & =R_{b}\left(t^{\varepsilon_{k-1}} ; n_{k}\right), \\
\mathcal{L}_{a}^{k}(t) & =L_{a}\left(t^{\varepsilon_{k-1}} ; n_{k}\right), & \mathcal{L}_{b}^{k}(t)=L_{b}\left(t^{\varepsilon_{k-1}} ; n_{k}\right) .
\end{array}
$$

Since $R_{a}(t ; n)=t^{(-1)^{n+1}} L_{b}\left(t^{-1} ; n\right)$ for any integer $n$,

$$
\mathcal{R}_{a}^{k}(t)=t^{\varepsilon_{k}} \mathcal{L}_{b}^{k}\left(t^{-1}\right) .
$$

For each $k=1,2, \ldots, r+1$, let $\mathcal{A}_{k}$ and $\mathcal{B}_{k}$ be Laurent polynomials in $\mathbb{Z}\left[t^{ \pm 1}, u^{ \pm 1}\right]$ defined by the following recursive relations:

$$
\begin{gathered}
\mathcal{A}_{1}=1 ; \\
\mathcal{B}_{1}=1 ; \\
\mathcal{A}_{k+1}=\mathcal{R}_{a}^{k}(t) \mathcal{A}_{k}+u \mathcal{R}_{b}^{k}(t) \mathcal{B}_{k} \\
\mathcal{B}_{k+1}=\mathcal{L}_{a}^{k}(t) \mathcal{A}_{k}+u \mathcal{L}_{b}^{k}(t) \mathcal{B}_{k}
\end{gathered}
$$

THEOREM 2.1 [9]. For given nonzero integers $n_{1}, n_{2}, \ldots, n_{r}$, let $\Delta^{k}(t, u)$ $(1 \leq k \leq r)$ be the Laurent polynomial in $\mathbb{Z}\left[t^{ \pm 1}, u^{ \pm 1}\right]$ given by

$$
\Delta^{k}(t, u)=\frac{u \mathcal{B}_{k+1}-\mathcal{A}_{k+1}}{u-1} .
$$


Then $\Delta^{k}(t, u)$ is the Alexander polynomial of a 2-bridge link $\vec{C}\left[\left[n_{1}, n_{2}, \ldots, n_{k}\right]\right]$ up to units and

$$
\begin{aligned}
& \Delta^{0}(t, u)=1, \\
& \Delta^{1}(t, u)=u \mathcal{L}_{b}^{1}(t)+\mathcal{R}_{a}^{1}(t), \\
& \Delta^{k}(t, u)=\left(u \mathcal{L}_{b}^{k}(t)+\mathcal{R}_{a}^{k}(t)\right) \Delta^{k-1}(t, u)+u \mathcal{T}^{k}(t)\left(\mathcal{R}_{b}^{k-1}(t)-\mathcal{L}_{b}^{k-1}(t)\right) \Delta^{k-2}(t, u) .
\end{aligned}
$$

THEOREM 2.2. Let $\Delta_{L}(t, u)$ be the Alexander polynomial of a 2-bridge link $L=$ $\vec{C}\left[\left[n_{1}, n_{2}, \ldots, n_{r}\right]\right]$ and let $\Delta_{L}(t, u)=\sum_{i=m}^{M} \phi_{i}(t) u^{i}$ with $\phi_{M}(t) \neq 0$ and $\phi_{m}(t) \neq 0$. Then $M=r, m=0$,

$$
\phi_{r}(t)= \pm t^{i} \mathcal{L}_{b}^{1}(t) \mathcal{L}_{b}^{2}(t) \cdots \mathcal{L}_{b}^{r}(t) \quad \text { and } \quad \phi_{0}(t)= \pm t^{j} \mathcal{R}_{a}^{1}(t) \mathcal{R}_{a}^{2}(t) \cdots \mathcal{R}_{a}^{r}(t)
$$

for some integers $i$ and $j$.

PRoOF. In [9], we proved that $M=r$ and $\phi_{r}(t)= \pm t^{i} \mathcal{L}_{b}^{1}(t) \mathcal{L}_{b}^{2}(t) \cdots \mathcal{L}_{b}^{r}(t)$ for some integer $i$ by showing that $\mathcal{L}_{b}^{k}(t) \neq 0$ for each $k=1,2, \ldots, r$ (see [9, Corollary 8].) From (2.1), it follows that $\mathcal{R}_{a}^{k}(t) \neq 0$ for each $k=1,2, \ldots, r$. By Theorem 2.1, we also have $m=0$ and $\phi_{0}(t)= \pm t^{j} \mathcal{R}_{a}^{1}(t) \mathcal{R}_{a}^{2}(t) \cdots \mathcal{R}_{a}^{r}(t)$. This completes the proof.

Let $\widehat{\Delta}_{L}(t)$ be the reduced Alexander polynomial of a link $L$. If $\widehat{\Delta}_{L}(t)=\sum_{i=0}^{M} a_{i} t^{i}$ $\left(a_{0} \neq 0 \neq a_{M}\right)$ up to units, then $M$ is called the degree of the reduced Alexander polynomial $\widehat{\Delta}_{L}(t)$ and we denote $M$ by $\operatorname{deg} \widehat{\Delta}_{L}(t)$. In 1971, Murasugi [13] showed that if $L=K \cup U$, where $U$ is unknotted, and $K^{(p)}$ is the $p$-periodic link in $S^{3}$ with $L$ as its quotient link, then

$$
\widehat{\Delta}_{K^{(p)}}(t)=\Delta_{K}(t) \prod_{i=1}^{p-1} \Delta_{L}\left(t, \omega^{i}\right)
$$

where $\omega$ is a primitive $p$ th root of unity and $\widehat{\Delta}_{K^{(p)}}(t)$ the reduced Alexander polynomial of $K^{(p)}$.

THEOREM 2.3. For given nonzero integers $n_{1}, n_{2}, \ldots, n_{r}(r \geq 1)$ and a positive integer $p \geq 2$, let $L^{(p)}$ be the p-periodic link with rational quotient $L=$ $\vec{C}\left[\left[n_{1}, n_{2}, \ldots, n_{r}\right]\right]$ and $\widehat{\Delta}_{L^{(p)}}(t)$ its reduced Alexander polynomial. Then

$$
\operatorname{deg} \widehat{\Delta}_{L^{(p)}}(t)=r(p-1) .
$$

Proof. Let $\Delta_{L}(t, u)=\sum_{i=0}^{r} \phi_{i}(t) u^{i}$ be the Alexander polynomial of $L=$ $\vec{C}\left[\left[n_{1}, n_{2}, \ldots, n_{r}\right]\right]=U_{1} \cup U_{2}$. By Theorem $2.2, \phi_{0}(t) \neq 0$ and $\phi_{r}(t) \neq 0$. Since every 2-bridge link is interchangeable [7], $\Delta_{L}(t, u)=\Delta_{L}(u, t)$ up to units. Since $U_{1}$ is unknotted, by (2.2),

$$
\widehat{\Delta}_{L^{(p)}}(t)=\prod_{i=1}^{p-1} \Delta_{L}\left(t, \omega^{i}\right)=\left.\prod_{i=1}^{p-1} \Delta_{L}\left(\omega^{i}, u\right)\right|_{u=t} .
$$




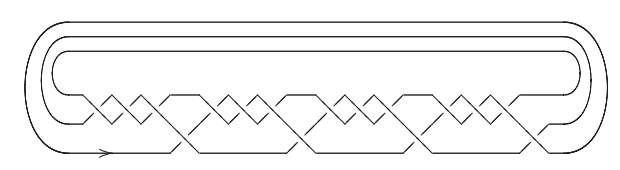

FIGURE 4. A 4-periodic knot which has no rational quotient.

Put $\widehat{\Delta}_{L^{(p)}}(t)=\sum_{i=0}^{r(p-1)} a_{i} t^{i}$. Then

$$
a_{0}=\prod_{i=1}^{p-1} \phi_{0}\left(\omega^{i}\right) \quad \text { and } \quad a_{r(p-1)}=\prod_{i=1}^{p-1} \phi_{r}\left(\omega^{i}\right) .
$$

From (2.1) and Theorem 2.2, we obtain that $\phi_{0}(t)= \pm t^{a} \phi_{r}\left(t^{-1}\right)$ for some integer $a$. In [9, Proof of Theorem 10], we showed that $a_{r(p-1)} \neq 0$. Since $\prod_{i=1}^{p-1} \omega^{i}=1$ and $\prod_{i=1}^{p-1} \phi_{r}\left(\omega^{-i}\right)=\prod_{i=1}^{p-1} \phi_{r}\left(\omega^{i}\right)$, we also see that

$$
a_{0}=\prod_{i=1}^{p-1} \phi_{0}\left(\omega^{i}\right)= \pm \prod_{i=1}^{p-1} \omega^{i a} \phi_{r}\left(\omega^{-i}\right)= \pm \prod_{i=1}^{p-1} \phi_{r}\left(\omega^{i}\right) \neq 0 .
$$

Hence, the degree of $\widehat{\Delta}_{L^{(p)}}(t)$ is $r(p-1)$. This completes the proof.

The following corollary is an immediate consequence of Theorem 2.3.

COROLlary 2.4. Let $L$ be any p-periodic link in $S^{3}$. If $L$ has a rational quotient, then

$$
\operatorname{deg} \widehat{\Delta}_{L}(t) \equiv 0 \bmod (p-1) .
$$

EXAmple 2.5. Let $K$ be an oriented knot in $S^{3}$ with a diagram as shown in Figure 4.

Then $K$ is a 4-periodic knot. Since the Alexander polynomial of $K$ is

$$
\begin{gathered}
\Delta_{K}(t)=1-t+t^{3}-t^{4}+t^{6}-t^{7}+t^{8}-t^{10}+t^{11}-t^{13}+t^{14}, \\
\operatorname{deg} \Delta_{K}(t) \not \equiv 0 \bmod 3 .
\end{gathered}
$$

Hence, $K$ does not have a rational quotient as a 4-periodic knot.

REMARK 2.6. Let $L$ be an oriented $p$-periodic link of $\mu$ components. If $L$ has a rational quotient $\vec{C}\left[\left[n_{1}, n_{2}, \ldots, n_{r}\right]\right]$, then $r=(1 /(p-1)) \operatorname{deg} \widehat{\Delta}_{L}(t)$ is an invariant of $L$.

Let $\sigma(L)$ denote the signature of an oriented link $L$ in $S^{3}$. In [18], Shinohara showed that for a link $L$ with $\widehat{\Delta}_{L}(t) \neq 0,|\sigma(L)| \leq \operatorname{deg} \widehat{\Delta}_{L}(t)$. By Theorem 2.3, we have the following result.

COROLlARY 2.7. For given nonzero integers $n_{1}, n_{2}, \ldots, n_{r}(r \geq 1)$ and a positive integer $p \geq 2$, let $L^{(p)}$ be the p-periodic link with rational quotient $L=$ $\vec{C}\left[\left[n_{1}, n_{2}, \ldots, n_{r}\right]\right]$. Then $\left|\sigma\left(L^{(p)}\right)\right| \leq r(p-1)$. 


\section{Various kinds of genus}

A Seifert surface for a link $L$ in $S^{3}$ is a compact, connected, and orientable surface $S$ in $S^{3}$ such that the boundary of $S$ is $L$, that is, $\partial(S)=L$. The minimum genus over all Seifert surfaces for $L$ is called the genus for $L$, denoted by $g(L)$. For a diagram $D$ of a link $L$, it is well known that a Seifert surface for $L$ can always be obtained from $D$ by applying Seifert's algorithm [17]. A Seifert surface for a link $L$ constructed via Seifert's algorithm for a diagram $D$ is called a canonical Seifert surface associated with the diagram $D$. Then the minimum genus over all canonical Seifert surfaces for $L$ is called the canonical genus for $L$, denoted by $g_{c}(L)$. A Seifert surface $S$ for a link $L$ is said to be free if the fundamental group $\pi_{1}\left(S^{3}-S\right)$ of the complement of the surface $S$ is a free group. The minimum genus over all free Seifert surfaces for $L$ is called the free genus for $L$, denoted by $g_{f}(L)$. The slice genus $g_{s}(L)$ of $L$ is the minimum genus over all Seifert surfaces for $L$ properly embedded in a 4-ball $B^{4}$. We first recall the following inequality.

TheOrem $3.1[2,17]$. Let $L$ be an oriented link in $S^{3}$ with $\mu$ components. Then

$$
\frac{1}{2}\left(\operatorname{deg} \widehat{\Delta}_{L}(t)-\mu+1\right) \leq g(L) .
$$

THEOREM 3.2. For given nonzero integers $n_{1}, n_{2}, \ldots, n_{r}(r \geq 1)$ and a positive integer $p \geq 2$, let $L^{(p)}$ be the p-periodic link with rational quotient $L=$ $\vec{C}\left[\left[n_{1}, n_{2}, \ldots, n_{r}\right]\right]$ and $D^{(p)}$ its canonical p-periodic diagram shown in Figure 3. Then the canonical Seifert surface associated with the diagram $D^{(p)}$ is a Seifert surface of minimal genus and

$$
\begin{aligned}
\frac{1}{2}\left(\operatorname{deg} \widehat{\Delta}_{L^{(p)}}(t)-\mu\left(L^{(p)}\right)+1\right) & =g\left(L^{(p)}\right)=g_{f}\left(L^{(p)}\right)=g_{c}\left(L^{(p)}\right) \\
& =\frac{1}{2}\left(r(p-1)-\mu\left(L^{(p)}\right)+1\right),
\end{aligned}
$$

where $\mu\left(L^{(p)}\right)$ denotes the number of components of $L^{(p)}$.

PROOF. Let $S$ be the canonical Seifert surface of $L^{(p)}$ associated to the canonical diagram $D^{(p)}$ and let $g(S)$ denote the genus of the surface $S$. Then

$$
2 g(S)+\mu\left(L^{(p)}\right)-1=c\left(D^{(p)}\right)-s\left(D^{(p)}\right)+1,
$$

where $c\left(D^{(p)}\right)$ denotes the number of crossings of $D^{(p)}$ and $s\left(D^{(p)}\right)$ denotes the number of Seifert circles of $D^{(p)}$. Observing the diagram $D^{(p)}$, we obtain

$$
c\left(D^{(p)}\right)=p \sum_{i=1}^{r}\left|n_{i}\right|, \quad s\left(D^{(p)}\right)=p \sum_{i=1}^{r}\left(\left|n_{i}\right|-1\right)+r+1 .
$$

Hence

$$
c\left(D^{(p)}\right)-s\left(D^{(p)}\right)+1=p \sum_{i=1}^{r}\left|n_{i}\right|-\left(p \sum_{i=1}^{r}\left(\left|n_{i}\right|-1\right)+r+1\right)+1=r(p-1) .
$$


By Theorem 3.1 and the equality (3.1) together with $g_{c}\left(L^{(p)}\right) \leq g(S)$,

$$
\begin{aligned}
\operatorname{deg} \widehat{\Delta}_{L^{(p)}}(t) & \leq 2 g\left(L^{(p)}\right)+\mu\left(L^{(p)}\right)-1 \leq 2 g_{c}\left(L^{(p)}\right)+\mu\left(L^{(p)}\right)-1 \\
& \leq c\left(D^{(p)}\right)-s\left(D^{(p)}\right)+1=r(p-1) .
\end{aligned}
$$

Hence, it follows from Theorem 2.3 and (1.2) that

$$
\begin{aligned}
\frac{1}{2}\left(\operatorname{deg} \widehat{\Delta}_{L^{(p)}}(t)-\mu\left(L^{(p)}\right)+1\right) & =g\left(L^{(p)}\right)=g_{f}\left(L^{(p)}\right)=g_{c}\left(L^{(p)}\right) \\
& =\frac{1}{2}\left(r(p-1)-\mu\left(L^{(p)}\right)+1\right)
\end{aligned}
$$

This completes the proof.

The following corollary is a direct consequence of Theorem 3.2.

Corollary 3.3. Let $L$ be an oriented p-periodic link of $\mu$ components. If $L$ has a rational quotient $\vec{C}\left[\left[n_{1}, n_{2}, \ldots, n_{r}\right]\right]$, then

$$
2 g(L) \equiv 1-\mu \quad \bmod (p-1) .
$$

ExAmple 3.4. Let $K$ be the 4-periodic knot in Example 2.5. Since $K$ is a positive knot and $\operatorname{deg} \Delta_{K}(t)=14$, the genus of $K$ is seven and, hence, $2 g \not \equiv 0 \bmod 3$.

THEOREM 3.5. Let $n_{1}, n_{2}, \ldots, n_{r}$ be given nonzero integers $(r \geq 1)$ and let $L^{(3)}$ be the 3-periodic link in $S^{3}$ with rational quotient $L=\vec{C}\left[\left[n_{1}, n_{2}, \ldots, n_{r}\right]\right]$. Suppose that $n_{1}, n_{2}, \ldots, n_{r}$ have the same sign and $\left|n_{i} n_{i+1} n_{i+2} n_{i+3}\right| \geq 2$ for each $i=$ $1,2, \ldots, r-3$. Then

$$
g_{s}\left(L^{(3)}\right)=g\left(L^{(3)}\right)=g_{f}\left(L^{(3)}\right)=g_{c}\left(L^{(3)}\right)=r+\frac{1-\mu\left(L^{(3)}\right)}{2} .
$$

PROOF. In [8], the authors showed that if $\left|n_{i} n_{i+1} n_{i+2} n_{i+3}\right| \geq 2$ for each $i=$ $1,2, \ldots, r-3$, then the signature $\sigma\left(L^{(3)}\right)$ of $L^{(3)}$ is given by

$$
\sigma\left(L^{(3)}\right)=-2 \sum_{i=1}^{r} \frac{n_{i}}{\left|n_{i}\right|}
$$

Since $n_{1}, n_{2}, \ldots, n_{r}$ have the same sign, $\sigma\left(L^{(3)}\right)= \pm 2 r$. On the other hand, Murasugi [12] proved that for a link $L,|\sigma(L)| \leq 2 g_{s}(L)+\mu(L)-1$. From this inequality, together with (1.2) and Theorem 3.2, we obtain

$$
r+\frac{1-\mu\left(L^{(3)}\right)}{2} \leq g_{s}\left(L^{(3)}\right) \leq g\left(L^{(3)}\right)=r+\frac{1-\mu\left(L^{(3)}\right)}{2} .
$$

This completes the proof. 
EXAMPLE 3.6.

(1) The 3-periodic knots with rational quotients $\vec{C}[[1,1,-1]]$ and $\vec{C}[[1,2]]$ are the knots $9_{47}$ and $9_{49}$ in Rolfsen's table [16], respectively. They are neither alternating links nor torus links. By Theorem 3.2, $g\left(9_{47}\right)=g_{f}\left(9_{47}\right)=g_{c}\left(9_{47}\right)=3$. However, $g_{s}\left(9_{47}\right)=1$ (see [7]). By Theorem 3.5, $g_{s}\left(9_{49}\right)=g\left(9_{49}\right)=g_{f}\left(9_{49}\right)=$ $g_{c}\left(9_{49}\right)=2$.

(2) Let $L^{(p)}$ be the $p$-periodic link in $S^{3}$ with rational quotient $L=\vec{C}\left[\left[n_{1}\right.\right.$, $\left.\left.n_{2}, \ldots, n_{r}\right]\right]$, where $n_{1}=n_{2}=\cdots=n_{r}=1$. Then $L^{(p)}$ is a torus link of type $(r+1, p)$. By Theorem 3.2, $g\left(L^{(p)}\right)=g_{f}\left(L^{(p)}\right)=g_{c}\left(L^{(p)}\right)=\frac{1}{2} r(p-1)$.

A Seifert surface $S$ for a $p$-periodic link $L$ in $S^{3}$ is said to be $p$-equivariant if it is invariant under an orientation-preserving auto-homeomorphism $h: S^{3} \rightarrow S^{3}$ which respects the period $p$ of $L$. In [3], Edmonds proved that if $K$ is a $p$-periodic knot, then there exists a $p$-equivariant minimal genus Seifert surface $S$ for $K$, that is, $g(S)=g(K)$. Note that if $K$ is a $p$-periodic knot with a $p$-periodic homeomorphism $h$, then a Seifert surface for the factor knot $K_{*}=K /\langle h\rangle$ can be lifted to give a $p$ equivariant Seifert surface $S$ for $K$. Conversely, any $p$-equivariant Seifert surface $S$ for $K$ gives a Seifert surface $S_{*}=S /\langle h\rangle$ for the factor knot $K_{*}$ of $K$.

THEOREM 3.7. Let $K$ be a p-periodic knot with rational quotient $\vec{C}\left[\left[n_{1}, n_{2}, \ldots\right.\right.$, $\left.n_{r}\right]$ ] $(p \geq 2)$. Let $S$ be any $p$-equivariant Seifert surface for $K$ with $g(S)=g(K)$ and let $h$ be an associated p-periodic homeomorphism. Then

$$
g\left(S_{*}\right)=\frac{p-1}{2 p}(r-m+1), \quad m \geq \ell, m \equiv \ell(\bmod 2),
$$

where

$$
\ell=1+\sum_{k=1}^{r}(-1)^{n_{1}+n_{2}+\cdots+n_{k}+k}
$$

and $m=|\operatorname{Fix}(h) \cap S|$, that is, the number of points of intersection of $S$ (or $S_{*}$ ) with Fix $(h)$. Furthermore, $S_{*}$ is a 2-disk if and only if $m=r+1$.

PROOF. We first observe that the restriction $h: S \rightarrow S_{*}$ is a $p$-fold branched cyclic covering branched along $\operatorname{Fix}(h) \cap S_{*}$. By Riemann-Hurwitz Formula, it follows $[3,14]$ that

$$
g(S)=p\left(g\left(S_{*}\right)\right)+\frac{(p-1)(m-1)}{2}, \quad m \geq \ell, m \equiv \ell(\bmod 2),
$$

where $\ell=\operatorname{lk}\left(K_{*}, \operatorname{Fix}(h)\right)$, that is, the linking number of $K_{*}$ and $\operatorname{Fix}(h)$. Since $K_{*} \cup \operatorname{Fix}(h)=\vec{C}\left[\left[n_{1}, n_{2}, \ldots, n_{r}\right]\right]$, it is easy to see from Figure 1 that

$$
\ell=1+\sum_{k=1}^{r}(-1)^{n_{1}+n_{2}+\cdots+n_{k}+k}
$$


Now, by Theorem 3.2, $g(S)=g(K)=r(p-1) / 2$ and hence (3.2) follows. Note that $S_{*}$ is a 2-disk if and only if $g\left(S_{*}\right)=0$. From (3.2), it is straightforward that $g\left(S_{*}\right)=0$ if and only if $m=r+1$. This completes the proof.

\section{Homogeneity}

Let $D$ be an oriented diagram of a link $L$ and $F$ its canonical Seifert surface associated with $D$. The spine of this surface is a graph, say $G$. The vertices of $G$ correspond to the disks in $F$ which span the Seifert circles of $D$; the edges of $G$ correspond to the crossings in $D$. Each edge in $G$ can be given a sign according to the sign of the corresponding crossing. A signed graph constructed from a diagram in this manner is called a Seifert graph. We assume that the links under consideration are nonsplit. This implies that the corresponding Seifert graphs are connected.

Let $G$ be any connected graph. An edge $e$ in $G$ is an isthmus if $G-e$ is disconnected. A vertex $v$ in $G$ is a cut vertex if $G-v$ is disconnected. Suppose that $G$ contains a cut vertex $v$ and let $G_{1}, \ldots, G_{n}$ be the connected components of $G-v$. Then the $n$ subgraphs $G_{1} \cup v, \ldots, G_{n} \cup v$ are obtained from $G$ by cutting $G$ at $v$. Cutting $G$ at each of its cut vertices produces a set of connected components, each one being a subgraph of $G$ containing no cut vertices. Such a component is called a block. A block of a Seifert graph is homogeneous if all of its edges have the same sign. A Seifert graph is homogeneous if each of its blocks is homogeneous. A diagram is homogeneous if its Seifert graph is homogeneous. A link is homogeneous if it has a homogeneous diagram [1].

Let $\chi(L)$ denote the maximal Euler characteristic over all Seifert surfaces for a link $L$. Then it is known [1] that for any link $L$ with $\mu$ components,

$$
1-\chi(L)=2 g(L)+\mu-1 .
$$

Let $\max \operatorname{deg}_{z} P_{L}(v, z)$ and $\min \operatorname{deg}_{v} P_{L}(v, z)$ denote the highest and the lowest degrees of $z$ in the Homfly polynomial $P_{L}(v, z)$ of a link $L$.

THEOREM 4.1. For given nonzero integers $n_{1}, n_{2}, \ldots, n_{r}(r \geq 1)$ and a positive integer $p \geq 2$, let $L^{(p)}$ be the p-periodic link with rational quotient $L=$ $\vec{C}\left[\left[n_{1}, n_{2}, \ldots, n_{r}\right]\right]$. Then:

(1) $L^{(p)}$ is a homogeneous link;

(2) $\quad \max \operatorname{deg}_{z} P_{L^{(p)}}(v, z)=1-\chi\left(L^{(p)}\right)=r(p-1)$;

(3) $\min \operatorname{deg}_{v} P_{L^{(p)}}(v, z) \leq 1-\chi\left(L^{(p)}\right)=r(p-1)$ with equality if and only if $L^{(p)}$ is positive; and

(4) if $\alpha(v)$ denotes the coefficient polynomial of $z^{r(p-1)}$ in $P_{L^{(p)}}(v, z)$ of a link $L^{(p)}$, then $\alpha(v)$ is a polynomial in $v$ and the coefficients of $\alpha(v)$ are all nonnegative or all nonpositive.

PROOF. For any nonzero integer $n$, let $G(n)$ be the signed graph as shown in Figure 5, where there are $p$ simple paths from $v_{1}$ to $v_{2}$ and each path consists of $|n|$ edges 


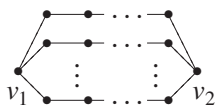

$G(n)$

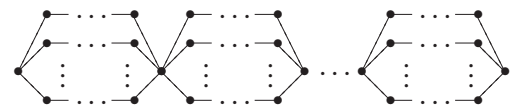

$G\left(n_{1}\right) * G\left(n_{2}\right) * \cdots * G\left(n_{r}\right)$

FIGURE 5. Seifert graph associated with $D^{(p)}$.

whose signs are all positive or all negative according to whether $n$ is positive or negative. For the integers $n_{1}, n_{2}, \ldots, n_{r}$, let $G\left(n_{1}\right) * G\left(n_{2}\right) * \cdots * G\left(n_{r}\right)$ be the block sum of $G\left(n_{1}\right), G\left(n_{2}\right), \ldots, G\left(n_{r}\right)$ as shown in Figure 5. Then it is easy to see that $G\left(n_{1}\right) * G\left(n_{2}\right) * \cdots * G\left(n_{r}\right)$ is the Seifert graph associated with the canonical diagram $D^{(p)}$ of $L^{(p)}$. Since all edges of $G\left(n_{i}\right)$ have the same sign, the diagram $D^{(p)}$ is homogeneous and, hence, $L^{(p)}$ is a homogeneous link. Combining [1, Theorem 4], Theorem 3.2, and the equality (4.1), we obtain the properties (2) and (3). From [1, Corollary 4.3], we obtain the property (4). This completes the proof.

REMARK 4.2. Let $L^{(p)}$ be the $p$-periodic link with rational quotient $\vec{C}\left[\left[n_{1}, n_{2}, \ldots\right.\right.$, $\left.n_{r}\right]$ and let $\nabla_{L^{(p)}}(z)$ be the Conway polynomial of the link $L^{(p)}$. From [1, Corollaries 5.2 and 5.3], [2, Corollary 7.6.5] and [9, Theorem 10], we also have following properties:

(1) $2 g\left(L^{(p)}\right)=\max \operatorname{deg} \nabla_{L^{(p)}}(z)-\mu\left(L^{(p)}\right)+1$ and the leading coefficient of $\nabla_{L^{(p)}}(z)$ is \pm 1 if and only if $n_{i}= \pm 1$ for all $i=1,2, \ldots, r$;

(2) $\quad L^{(p)}$ does not have a special homogeneous diagram $(r \geq 2)$ and it is fibered if and only if the leading coefficient of $\nabla_{L^{(p)}}(z)$ is \pm 1 .

\section{Acknowledgements}

The authors would like to thank Akio Kawauchi for valuable comments. This work was supported for two years by a Pusan National University Research Grant.

\section{References}

[1] P. R. Cromwell, 'Homogeneous links', J. London Math. Soc. 39 (1989), 535-552.

[2] Knots and Links (Cambridge University Press, Cambridge, 2004).

[3] A. Edmonds, 'Least area Seifert surfaces and periodic knots', Topol. Appl. 18 (1984), 109-113.

[4] F. Frankel and L. Pontrjagin, 'Ein Knotensatz mit Anwendung auf die Dimensionstheorie', Math. Ann. 102 (1930), 785-789.

[5] H. M. Hilden, M. T. Lozano and J. M. Montesinos-Amilibia, 'On the character variety of periodic knots and links', Math. Proc. Camb. Phil. Soc. 129 (2000), 477-490.

[6] H. J. Jang, S. Y. Lee and M. Seo, 'Casson knot invariants of periodic knots with rational quotients', J. Knot Theory Ramifications 16 (2007), 439-460.

[7] A. Kawauchi, A Survey of Knot Theory (Birkhäuser, Basel, 1996).

[8] S. Y. Lee, M.-S. Park and M. Seo, 'The Seifert matrices of periodic links with rational quotients', Kyungpook Math. J. 47 (2007), 295-309. 
[9] S. Y. Lee and M. Seo, 'Recurrence formulas for the Alexander polynomials of 2-bridge links and their covering links', J. Knot Theory Ramifications 15 (2006), 179-203.

[10] K. Murasugi, 'On the genus of the alternating knot I', J. Math. Soc. Japan 10 (1958), 94-105.

[11] - 'On the genus of the alternating knot II', J. Math. Soc. Japan 10 (1958), 235-248.

[12] - 'On a certain numerical invariant of link types', Trans. Amer. Math. Soc. 117 (1965), $387-422$.

[13] _ 'On periodic knots', Comm. Math. Helv. 35 (1971), 529-537.

[14] S. Naik, 'Periodicity, genera and Alexander polynomials of knots', Pacific J. Math. 166 (1994), 357-371.

[15] T. Nakamura, 'On canonical genus of fibered knot', J. Knot Theory Ramifications 11 (2002), 341-352.

[16] D. Rolfsen, Knots and Links (AMS/Chelsea, New York, 2003).

[17] H. Seifert, 'Über das Geschlecht von Knoten', Math. Ann. 110 (1936), 571-592.

[18] Y. Shinohara, 'On the signature of knots and links', Trans. Amer. Math. Soc. 156 (1971), 273-285.

[19] J. J. Tripp, 'The canonical genus of whitehead doubles of a family torus knots', J. Knot Theory Ramifications 11 (2002), 1233-1242.

\author{
SANG YOUL LEE, Department of Mathematics, Pusan National University, \\ Pusan 609-735, Korea \\ e-mail: sangyoul@pusan.ac.kr
}

MYOUNGSOO SEO, Department of Mathematics, Kyungpook National University, Daegu 702-701, Korea

e-mail: myseo@knu.ac.kr 